\title{
BMJ Open Validation of two case definitions to identify pressure ulcers using hospital administrative data
}

\author{
Chester Ho, ${ }^{1}$ Jason Jiang, ${ }^{2}$ Cathy A Eastwood, ${ }^{3}$ Holly Wong, ${ }^{3}$ Brittany Weaver, ${ }^{4}$ \\ Hude Quan ${ }^{3}$
}

To cite: Ho C, Jiang J, Eastwood CA, et al. Validation of two case definitions to identify pressure ulcers using hospital administrative data. BMJ Open 2017;7:e016438. doi:10.1136/ bmjopen-2017-016438

- Prepublication history and additional material for this paper are available online. To view these files please visit the journal online (http://dx.doi. org/10.1136/bmjopen-2017016438).

Received 17 February 2017 Revised 16 June 2017 Accepted 3 July 2017

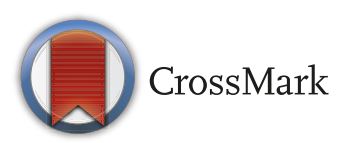

${ }^{1}$ Division of Physical Medicine \& Rehabilitation, Department of Clinical Neurosciences, Cumming School of Medicine, University of Calgary, Calgary, Canada

${ }^{2}$ Analytics, Alberta Health Services, Calgary, Canada ${ }^{3}$ Department of Community Health Sciences, Cumming School of Medicine, University of Calgary, Calgary, Canada

${ }^{4}$ Family Medicine, University of British Columbia Faculty of Medicine, Canada

Correspondence to Dr Chester Ho; chester.ho@albertahealthse rvices.ca

\section{ABSTRACT}

Objective Pressure ulcer development is a quality of care indicator, as pressure ulcers are potentially preventable. Yet pressure ulcer is a leading cause of morbidity, discomfort and additional healthcare costs for inpatients. Methods are lacking for accurate surveillance of pressure ulcer in hospitals to track occurrences and evaluate care improvement strategies. The main study aim was to validate hospital discharge abstract database (DAD) in recording pressure ulcers against nursing consult reports, and to calculate prevalence of pressure ulcers in Alberta, Canada in DAD. We hypothesised that a more inclusive case definition for pressure ulcers would enhance validity of cases identified in administrative data for research and quality improvement purposes.

Setting A cohort of patients with pressure ulcers were identified from enterostomal (ET) nursing consult documents at a large university hospital in 2011.

Participants There were 1217 patients with pressure ulcers in ET nursing documentation that were linked to a corresponding record in DAD to validate DAD for correct and accurate identification of pressure ulcer occurrence, using two case definitions for pressure ulcer.

Results Using pressure ulcer definition 1 (7 codes), prevalence was $1.4 \%$, and using definition 2 (29 codes), prevalence was $4.2 \%$ after adjusting for misclassifications. The results were lower than expected. Definition 1 sensitivity was $27.7 \%$ and specificity was $98.8 \%$, while definition 2 sensitivity was $32.8 \%$ and specificity was 95.9\%. Pressure ulcer in both DAD and ET consultation increased with age, number of comorbidities and length of stay.

Conclusion DAD underestimate pressure ulcer prevalence. Since various codes are used to record pressure ulcers in DAD, the case definition with more codes captures more pressure ulcer cases, and may be useful for monitoring facility trends. However, low sensitivity suggests that this data source may not be accurate for determining overall prevalence, and should be cautiously compared with other prevalence studies.

\section{BACKGROUND}

The development of a pressure ulcer (also called pressure injury) has been considered an indicator for quality of care, as pressure ulcers are potentially preventable, a leading cause of morbidity for inpatients, ${ }^{1}$ and is a

\section{Strengths and limitations of this study}

- We examined a large sample of patients for prevalence of pressure ulcers against enterostomal specialist consult descriptions to derive and validate a case definition in disease classification codes.

- This study adds evidence of underestimation of pressure ulcer prevalence in administrative hospital data that could potentially be improved with more specific documentation and coding.

- A potential bias may be present from use of enterostomal nurse consultation notes that included mostly severe (stage III, IV and unstageable) pressure ulcers.

- The descriptive textual database created in this study is the foundation for future work on autodetection of pressure ulcers in hospital records, to enhance the accuracy of prevalence estimates.

cause of substantial discomfort, prolonged hospitalisations, additional healthcare costs and, in some cases, death. ${ }^{1}$ The National Pressure Ulcer Advisory Panel defines a pressure ulcer as 'a localised injury to the skin and/or underlying tissue over a bony prominence, as a result of pressure, or pressure in combination with shear'. ${ }^{2}$ The severity of pressure ulcers can vary from skin erythema to full-thickness tissue loss, with damage extending to the muscle and bone. ${ }^{2}$ It is estimated that pressure ulcers affect 250000 to 500000 patients, with an annual prevalence of $21 \%-26 \%$ in healthcare institutions in Canada. ${ }^{34}$ In the USA, pressure ulcer prevalence ranged from $10 \%$ to $18 \%$ in general acute care, $2.3 \%$ to $28 \%$ in long-term care and $0 \%$ to $29 \%$ in home care between 1990 and $2000 .{ }^{5}$ In a recent review of international studies, pressure ulcer prevalence in acute care was estimated at $6 \%$ to $18.5 \% .{ }^{6}$ As more severe cases require intensive treatments, have a prolonged healing time and are associated with higher incidence of complications, ${ }^{7}$ the estimated cost of treatment varies from $£ 1214$ to $£ 14108$ per case in the $\mathrm{UK}^{7}$ and 
US $\$ 124327$ to US $\$ 129248$ in the USA for stage IV pressure ulcers. ${ }^{8}$ In Canada, the estimated average monthly cost of pressure ulcer management among individuals with a spinal cord injury was $\$ 4475 \mathrm{CDN}$ in $2010 .^{9}$

To date, information about pressure ulcers has been primarily obtained through cross-sectional surveys, incident reports and chart reviews. Surveys, incident reports and reporting systems, such as the National Health Service National Safety Thermometer in the UK, involve voluntary reporting which can result in inaccurate and under-reported data. ${ }^{10}$ The medical chart has been considered the 'reference standard' as a source of research and quality improvement data due to the clinical information it contains. Thus, retrospective reviews of medical records have been undertaken to identify prevalence and incidence of pressure ulcers as well as patient characteristics and associated risk factors among various patient populations, ${ }^{41-24}$ evaluate preventive and management strategies ${ }^{25-29}$ and evaluate the Braden scale in assessing risk for pressure ulcer development. ${ }^{30}$ Chart reviews and prospective studies involving physical assessment are costly and time-consuming; thus, other data sources are needed for surveillance.

Taking into account these barriers, administrative health data have been used as an alternate data source for pressure ulcer epidemiology and surveillance. Administrative health data are routinely collected and population-based and can offer a more comprehensive picture of a large population. ${ }^{31}$ These data are collected by certified coding specialists (2-year Health Information Management diploma) who extract information about conditions and procedures from hybrid paper and electronic inpatient health records. They then assign WHO International Disease Classification codes 10th version, Canada (ICD-10-CA). As administrative health data are collected for purposes other than research, it may be limited in its generalisability, continuity, accuracy and completeness.

To date, several studies have used administrative health data to determine adverse events. ${ }^{32-37}$ Some studies in the USA have used the national Medicare Patient Safety Monitoring System database to determine the national and state incidence of hospital-acquired pressure ulcers, ${ }^{38}$ and the 2003 Nationwide Inpatient Sample database to identify risk factors for pressure ulcer development among African Americans. ${ }^{39}$ One study examined trends in the prevalence and localisation of pressure ulcers and comorbidities among hospitalised patients in Germany from 2005 to 2011 by using nationally collected hospital data. ${ }^{40}$ While administrative health data have been found to be valid in one study for identifying adverse events, including pressure ulcers, among older hospitalised patients, ${ }^{41}$ findings from other studies indicated that administrative health data may not be reliable for examining pressure ulcer development among hospitalised patients, ${ }^{42} 43$ and occurrence in long-term care. ${ }^{44}$

In consideration of the paucity and discrepancies in the literature, the purpose of our study was to validate
Canadian administrative health data, also called hospital discharge abstract database (DAD), for determining if DAD could be used for pressure ulcer epidemiological studies and surveillance of pressure ulcers for quality improvement. However, the ICD-10-CA codes in DAD include those specific to pressure ulcer only, as well as those which refer to more generic skin conditions and may indeed be used for the coding of pressure ulcer. There is currently no standard for coding pressure ulcer in DAD. Our three specific objectives were (1) to validate $\mathrm{DAD}$ in recording pressure ulcer, (2) to calculate prevalence of pressure ulcer using DAD and (3) to adjust the prevalence to account for DAD validity. We hypothesised that a more inclusive case definition for pressure ulcers would enhance validity of cases identified in administrative data for research and quality improvement purposes.

\section{MATERIALS AND METHODS Study design}

Our study team completed a cross-sectional retrospective analysis of enterostomal therapy (ET) nursing documentation and DAD independently to identify every pressure ulcer occurrence documented in each source for the year of 2011 (period prevalence). The DAD collect information from the patient's chart for each hospital discharge through professional coders. Validation was achieved by comparing it to the 'reference standard' of data abstracted from wound care documentation by ET nurses. Unlike the existing literature, our study draws on Canadian data and validates $\mathrm{DAD}$ by comparing it with documentation by ET nurses, which is likely to be the most accurate 'reference standard' of chart recording on pressure ulcer management.

ET nurses are registered nurses who have received additional training to specialise in the provision of wound (including pressure ulcer), ostomy and continence care. ET nurses are wound care specialists who are often present in larger hospitals to consult for treatment guidelines. ET nurse consults occur for more advanced pressure ulcers and, therefore, more detailed documentation is typically present. Each occurrence from the ET nursing documentation was linked to its corresponding record in DAD through personal unique identifier (personal health number), in order to validate DAD for correct and accurate identification of pressure ulcer occurrence. We validated and compared two DAD coding definitions (box) and calculated sensitivity, specificity, positive predictive value (PPV) and negative predictive value $(\mathrm{NPV})$.

\section{Setting and study population}

This study was conducted at a tertiary referral university hospital situated in a large city in Canada. It is one of the country's largest medical facilities with over 1000 acute inpatient beds. 
Box International Classification of Diseases 10th Version, Canada (ICD-10-CA) Codes

\section{Definition 1}

L89 Decubitus (pressure) ulcer and pressure area

L89.0 Stage I decubitus ulcer and pressure area

L89.1 Stage II decubitus (pressure) ulcer

L89.2 Stage III decubitus (pressure) ulcer

L89.3 Stage IV decubitus (pressure) ulcer

L89.80 Decubitus (pressure) ulcer, unstageable

L89.9 Decubitus ulcer and pressure area, unspecified

\section{Definition 2}

L89 Decubitus (pressure) ulcer and pressure area

L89.0 Stage I decubitus ulcer and pressure area

L89.1 Stage II decubitus (pressure) ulcer

L89.2 Stage III decubitus (pressure) ulcer

L89.3 Stage IV decubitus (pressure) ulcer

L89.80 Decubitus (pressure) ulcer, unstageable

L89.9 Decubitus ulcer and pressure area, unspecified

L97 Ulcer of lower limb, not elsewhere classified

L98.4 Chronic ulcer of skin, not elsewhere classified

S00 Superficial injury of head

S01 Open wound of head

S10 Superficial injury of neck

S11 Open wound of neck

S20 Superficial injury of thorax

S21 Open wound of thorax

S30 Superficial injury of abdomen, lower back and pelvis

S31 Open wound of abdomen, lower back and pelvis7.4

S40 Superficial injury of shoulder and upper arm

S41 Open wound of shoulder and upper arm

S50 Superficial injury of forearm

S51 Open wound of forearm

S60 Superficial injury of wrist and hand

S61 Open wound of wrist and hand

S70 Superficial injury of hip and thigh

S71 Open wound of hip and thigh

S80 Superficial injury of lower leg

S81 Open wound of lower leg

S90 Superficial injury of ankle and foot

s91 Open wound of ankle and foot

\section{Data sources}

\section{Enterostomal nurse documentation}

We obtained nursing documentation on inpatients that received a consultation with an ET nurse (here on referred to as 'ET nurse consults') in the year of 2011. Patients with pressure ulcers may be referred for ET nurse consults in our facility. Therefore, ET nurse consults were chosen as the most accurate way to identify actual pressure ulcer occurrences in the hospital. These would include but are not limited to: pressure ulcers, diabetic foot ulcers, venous leg ulcers and other skin conditions.

All of these consult requests are stored in a binder and maintained by the ET nursing team. Every ET nurse consult from the binder from 2011 was included in our study.

A medical student was trained by the principal investigator, who has expertise on pressure ulcer, on how to review the consult documentation to determine whether the wound(s) of interest was a pressure ulcer. The medical student analysed each consult to identify those that described a pressure ulcer. Documentation from follow-up consults and duplicate consults were treated as one consult. For consult documentation that did not clearly indicate whether a pressure ulcer was being treated, the respective medical chart was obtained and reviewed by the medical student and principal investigator to determine whether a pressure ulcer was being treated. Consultations regarding wounds unrelated to pressure ulceration were excluded from our sample OR categorised as not pressure ulcer-related.

Included consultations concerning a pressure ulcer were then categorised by the location of the pressure ulcer. The anatomic categories included sacral ulcers, leg ulcers, ankle ulcers, heel ulcers, foot ulcers and other ulcers. Patients with pressure ulcer in multiple locations had each ulcer entered separately in our database.

A trained medical student extracted the following information: patient hospital identification code, whether the consultation was pressure-ulcer related, location (s) of the pressure ulcer(s) and their severity. This information was used as our 'reference standard' to verify the existence of pressure ulcers among the patients included in the study, which were then compared with the DAD of the corresponding patients.

\section{Discharge abstract data}

This hospital's DAD were obtained from the data repository managed by the region's single health authority. This repository integrates data from multiple source systems, linking them as well as aggregating them to support measurement, reporting and survey purposes. For each discharge, DAD abstract and summarise demographic, clinical and administrative information such as date and time of admission, date of discharge, length of stay and a maximum of 50 diagnostic codes (ICD-10-CA) and 20 procedure codes. To identify cases of pressure ulcer(s) in DAD for the year 2011, we used an ICD-10 coding definition that was developed by a group of wound care clinicians, ET nurses and nurse researcher. The process involved independent review of the ICD-10 codes by each of these people to identify codes which they considered to be related to pressure ulcers. Then, a meeting was held to discuss the identified codes for pressure ulcers. Ultimately, we decided to test two coding definitions. One definition is more specific (with only L89 codes) and should yield greater detection accuracy. The second definition is more inclusive (with L89 and other non-specific codes for wounds) and will likely capture a larger number of cases. The ICD-10 codes used for both definitions included in our study are presented in box.

\section{Linkage}

Consultation data were linked with DAD using each patient's personal health number. To ensure that we are linking each consultation to the correct admission, we 
decided to only link consultations with the admission during which the consultation occurs. In other words, our linkage criteria specified that the consultation date must fall within the admission and discharge dates.

\section{Statistical analysis}

Before conducing validation, we identified pressure ulcer cases in DAD. Using the two previously described ICD-10 coding definitions, we identified pressure ulcer cases in discharge data for all admissions at the large university hospital in 2011, and calculated a pressure ulcer prevalence using each definition.

The existence of pressure ulcers was compared between the two databases, and we calculated measures of sensitivity, specificity, PPV and NPV for each definition. This allows us to compare the predictive accuracy of each definition. We then used sensitivity and PPV to adjust our preliminary prevalence. We adjusted overall prevalence and each stratified subgroup. Details of our adjustment method can be found in online supplementary appendix A. This adjusted prevalence represents what the prevalence would have been had there been perfect sensitivity and PPV. We decided to only adjust using sensitivity and PPV, due to the fact that we had a low number of positive cases. Adjustment by sensitivity, specificity, PPV and NPV would have yielded nonsensical results.

All data analyses were conducted using SAS V.9.3. SAS is an analytical software that is well suited to working with large databases.

\section{RESULTS}

Our consultation data contained 1575 ET nurse consults. Of these, 318 were found to be duplicates and two patients were under the age of 18 . These records were removed. Thirty-eight were cases of diabetic ulcers. It was decided by the study team that diabetic ulcers would not be included and these cases were also removed. The remaining sample included 1217 unique ET nurse consults which were linked to their matching admission in DAD (figure 1).

Comparing pressure ulcer information in DAD against that in consultation, we calculated sensitivity of $27.7 \%$, specificity of $98.8 \%$, PPV of $91.7 \%$ and NPV of $73.9 \%$ for definition 1, and a sensitivity of $32.8 \%$, a specificity of $95.9 \%$, a PPV of $79.3 \%$ and an NPV of $74.6 \%$ for definition 2 (table 1). Regardless of definition, validity of ICD coding for pressure ulcers was relatively stable across groups by age and sex. However, validity of ICD coding improved with increased length of stay.

Table 2 shows the prevalence and adjusted prevalence of pressure ulcers in DAD, stratified by age, sex and length of stay using both definitions. We see that the adjusted prevalence is approximately double overall, and for most stratifications. The stratified prevalence indicates males were more likely to have pressure ulcers compared with females, and that longer length of stay is correlated with higher pressure ulcer prevalence.

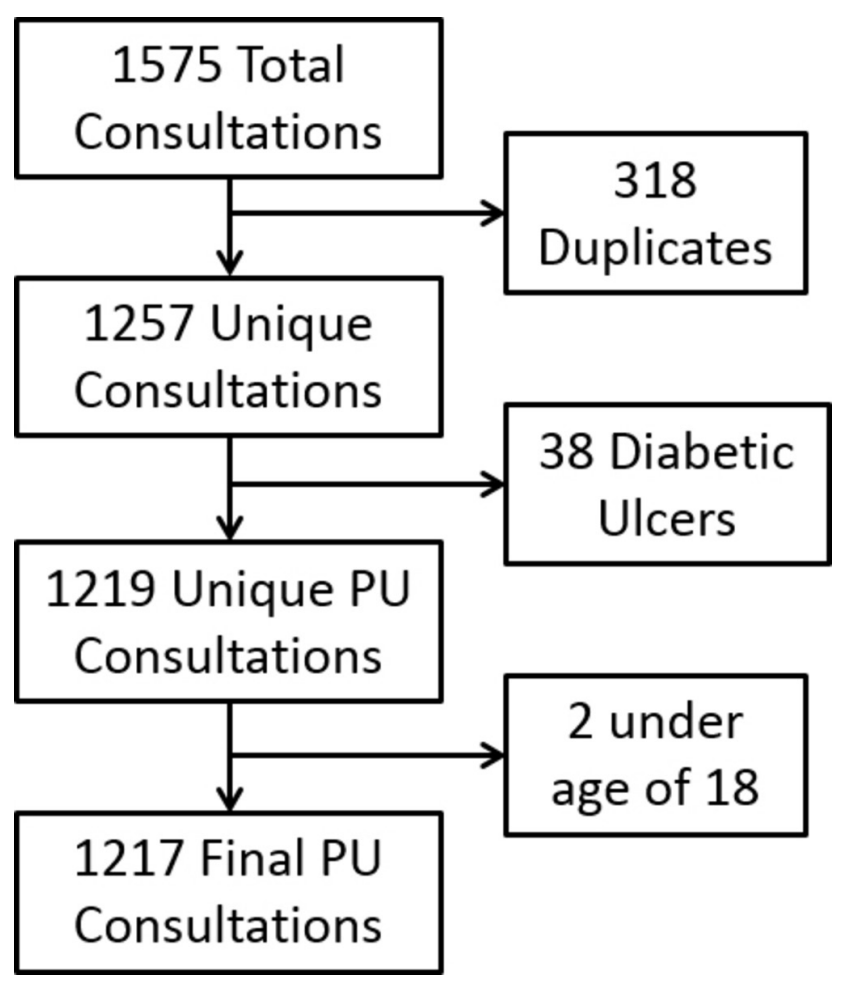

Figure 1 Flow diagram of pressure ulcer case selection from enterostomal therapy nurse consultations. PU, pressure ulcer.

Table 3 indicates pressure ulcer coding against age, sex, number of comorbidities and length of stay in both DAD and consultation data. Based on OR, we see that while pressure ulcer coding in DAD is similar between age groups, older patients are more likely to be coded with pressure ulcers in the nursing consultations. Further, males are significantly more likely to be coded with pressure ulcers in DAD, whereas the difference between the sexes is minimal in the consultations. For both DAD and nursing consultations, coding of pressure ulcers increased as the patient presents a greater number of comorbidities and a longer length of stay.

Lastly, table 4 lists the eight most common locations of pressure ulcers from the nursing consultations. Of note, there were a wide range of pressure ulcer locations. The eight most common locations only accounted for $32.6 \%$ of all pressure ulcer cases. The most common location by far was trochanteric pressure ulcer.

\section{DISCUSSION}

The objective of our study was to determine whether routinely collected administrative health data could be used for improved measurement of pressure ulcers. Since there is no standard code definition for pressure ulcer, we used expert consensus to determine two coding definitions from administrative health data to estimate the prevalence. Next, we validated these definitions against wound care documentation by ET nurses. After adjusting for misclassifications of pressure ulcers, using definition 1, we found 
Table 1 Validity of administrative health data for pressure ulcers using consultation as reference standard ( $\mathrm{n}=1217$ )

\begin{tabular}{|c|c|c|c|c|c|}
\hline & & Sensitivity & Specificity & PPV & NPV \\
\hline \multicolumn{6}{|c|}{ Definition 1 (7 ICD-10-CA codes) } \\
\hline Overall & & $27.7 \%$ & $98.8 \%$ & $91.7 \%$ & $73.9 \%$ \\
\hline \multirow{2}{*}{ Age } & $35-64$ & $32.0 \%$ & $98.5 \%$ & $88.9 \%$ & $79.8 \%$ \\
\hline & 65 and over & $25.4 \%$ & $99.4 \%$ & $96.7 \%$ & $67.4 \%$ \\
\hline Sex & Female & $26.1 \%$ & $99.2 \%$ & $93.9 \%$ & $74.6 \%$ \\
\hline \multirow[t]{4}{*}{ Length of stay } & $1-30$ days & $19.5 \%$ & $99.7 \%$ & $95.4 \%$ & $78.4 \%$ \\
\hline & 31-90 days & $35.9 \%$ & $96.9 \%$ & $90.2 \%$ & $65.7 \%$ \\
\hline & $91-180$ days & $40.5 \%$ & $90.0 \%$ & $83.3 \%$ & $55.1 \%$ \\
\hline & 181 days or more & $36.4 \%$ & $100.0 \%$ & $100.0 \%$ & $51.7 \%$ \\
\hline \multirow[t]{3}{*}{ Age } & $18-34$ & $40.0 \%$ & $92.2 \%$ & $60.0 \%$ & $83.9 \%$ \\
\hline & $35-64$ & $39.3 \%$ & $95.6 \%$ & $77.6 \%$ & $81.2 \%$ \\
\hline & 65 and over & $28.0 \%$ & $96.4 \%$ & $83.3 \%$ & $67.5 \%$ \\
\hline \multirow[t]{2}{*}{ Sex } & Male & $34.4 \%$ & $95.7 \%$ & $80.0 \%$ & $74.3 \%$ \\
\hline & Female & $30.7 \%$ & $96.1 \%$ & $78.3 \%$ & $75.1 \%$ \\
\hline \multirow[t]{4}{*}{ Length of stay } & $1-30$ days & $23.8 \%$ & $97.2 \%$ & $74.6 \%$ & $78.8 \%$ \\
\hline & $31-90$ days & $41.4 \%$ & $92.6 \%$ & $81.5 \%$ & $66.7 \%$ \\
\hline & 91-180 days & $46.0 \%$ & $90.0 \%$ & $85.0 \%$ & $57.5 \%$ \\
\hline & 181 days or more & $45.5 \%$ & $86.7 \%$ & $83.3 \%$ & $52.0 \%$ \\
\hline
\end{tabular}

ICD-10-CA, International Disease Classification codes 10th version, Canada; NPV, negative predictive value; PPV, positive predictive value.

prevalence of $1.4 \%$, and using definition 2 , we found a prevalence of $4.2 \%$. Definition 1 had a sensitivity of $27.7 \%$ and a specificity of $98.8 \%$, while definition 2 had a sensitivity of $32.8 \%$ and a specificity of $95.9 \%$. This means that the more specific definition 1 more accurately defines cases at the expense of missing some positive cases. Further, definition 2 includes more positive cases at the risk of a larger number of false positives. Both definitions show low sensitivity and high specificity. These findings indicate that administrative health data underestimate the prevalence of pressure ulcers. Although the more inclusive definition 2 had slightly higher sensitivity compared with definition 1 , the low sensitivity reflects the potential for the high frequency of missed pressure ulcer cases.

Compared with other studies of pressure ulcer prevalence, using the same set of ICD-10 codes (definition 1 ), our finding of $0.4 \%$ for unadjusted prevalence is lower than another Canadian study that reported 3.5\% cross-sectional prevalence, which can produce an overestimation. ${ }^{4}$ As well, our prevalence of $0.4 \%$ is substantially low relative to previous studies described above. ${ }^{35}$ This can be attributed to several factors: the assumption that ET nurse consults likely included mostly the more severe (stage III, IV and unstageable) pressure ulcers while other studies included pressure ulcers of all severities, there were differences in the population(s) studied and there were different methods and data sources used. For example, the review by Woodbury et al found an estimated pressure ulcer prevalence rate of $26 \%$ based on studies between 1990 and 2003 across a range of healthcare settings in Canada, including mostly pressure ulcers determined by clinical data versus coded data. ${ }^{3}$ Similarly, Cuddigan et al found prevalence rates ranging from $10 \%$ to $18 \%$ from analysing prevalence data spanning a decade (1990-2000) and across care settings, again, based on mostly direct examination or chart review data. ${ }^{5}$ Our prevalence rate was based on administrative health data from one tertiary acute care institution over 1 year. With such wide variations in care settings and time frames, these differences in prevalence rates are not surprising.

Another explanation to account for the underestimated prevalence rate is the inherent nature of the administrative health data. Our finding of either $27.7 \%$ or $32.8 \%$ sensitivity indicates a large proportion of pressure ulcers are not captured in the administrative health data. While this is a high percentage of missing cases, it is not an unreasonable finding. ${ }^{34}$ One of the primary purposes of collecting administrative health data is to inform resource utilisation, thus database administrators in Canada use predefined classification codes to register conditions that contribute to the length of stay in the hospital. Thus, less severe pressure ulcers, namely those identified as stage 
Table 2 Prevalence and adjusted prevalence of pressure ulcers in discharge abstract database (DAD)

\begin{tabular}{|c|c|c|c|c|c|c|}
\hline & \multicolumn{2}{|c|}{ Coded in DAD } & \multicolumn{3}{|c|}{ Adjusted based on sensitivity and PPV } \\
\hline & & Number of cases & Prevalence & Number of cases & Adjusted prevalence & Sample size \\
\hline \multicolumn{7}{|l|}{ Definition 1} \\
\hline Overall & & 163 & $0.4 \%$ & 539 & $1.4 \%$ & 38820 \\
\hline \multirow[t]{3}{*}{ Age } & $18-34$ & 9 & $0.1 \%$ & 27 & $0.3 \%$ & 8667 \\
\hline & $35-64$ & 69 & $0.4 \%$ & 192 & $1.1 \%$ & 16781 \\
\hline & 65 and over & 85 & $0.6 \%$ & 324 & $2.4 \%$ & 13372 \\
\hline \multirow[t]{2}{*}{ Sex } & Male & 100 & $0.6 \%$ & 311 & $1.9 \%$ & 16724 \\
\hline & Female & 63 & $0.3 \%$ & 227 & $1.0 \%$ & 22096 \\
\hline \multirow[t]{4}{*}{ Length of stay } & $0-30$ days & 57 & $0.2 \%$ & 279 & $0.8 \%$ & 36752 \\
\hline & $31-90$ days & 74 & $4.3 \%$ & 186 & $10.8 \%$ & 1724 \\
\hline & $91-180$ days & 22 & $8.5 \%$ & 45 & $17.5 \%$ & 258 \\
\hline & 181 days or more & 10 & $11.6 \%$ & 27 & $31.9 \%$ & 86 \\
\hline \multicolumn{7}{|l|}{ Definition 2} \\
\hline Overall & & 670 & $1.7 \%$ & 1620 & $4.2 \%$ & 38820 \\
\hline \multirow[t]{3}{*}{ Age } & $18-34$ & 179 & $2.1 \%$ & 269 & $3.1 \%$ & 8667 \\
\hline & $35-64$ & 272 & $1.6 \%$ & 537 & $3.2 \%$ & 16781 \\
\hline & 65 and over & 219 & $1.6 \%$ & 652 & $4.9 \%$ & 13372 \\
\hline \multirow[t]{2}{*}{ Sex } & Male & 437 & $2.6 \%$ & 1016 & $6.1 \%$ & 16724 \\
\hline & Female & 233 & $1.1 \%$ & 594 & $2.7 \%$ & 22096 \\
\hline \multirow[t]{4}{*}{ Length of stay } & $0-30$ days & 504 & $1.4 \%$ & 1580 & $4.3 \%$ & 36752 \\
\hline & $31-90$ days & 117 & $6.8 \%$ & 230 & $13.4 \%$ & 1724 \\
\hline & $91-180$ days & 31 & $12.0 \%$ & 57 & $22.2 \%$ & 258 \\
\hline & 181 days or more & 18 & $20.9 \%$ & 33 & $38.3 \%$ & 86 \\
\hline
\end{tabular}

PPV, positive predictive value.

I and II, are unlikely to be abstracted into the database. Rather, the low sensitivities mostly reflect the occurrence of stage III, IV and unstageable pressure ulcers, the most severe cases that would indeed lead to hospitalisation or contribute to the length of stay. While an adjusted prevalence rate of $4.2 \%$ is relatively low when compared with the overall prevalence of all stages of pressure ulcers across various healthcare settings, ${ }^{3}$ it is comparable to the prevalence rates of severe pressure ulcers found in acute care facilities in other studies. ${ }^{45} 46$

A unique feature of our study was defining the pressure ulcer cohort by ET nurse consults. This method ensured that clear pressure ulcer cases were included. As a result, there may have been more medical documentation in the health record on wound description and treatment on these clear cases of pressure ulcers. Therefore, we suspect that there may have been a greater chance for ICD coding abstractors to detect the pressure ulcer when ET nurses are consulted for the more severe ulcerations. That being said, less severe pressure ulcer cases may have been missed when no formal consultation is made, which are often only recorded by nurses and not seen by ICD coding abstractors.

Administrative health data have been used for various purposes, including chronic disease surveillance, quality of care assessments and population health reports. This data source has been found to be well coded for hypertension $^{47}$ and diabetes. ${ }^{48}$ Similarly for pressure ulcer, administrative data analysis may serve to identify the magnitude of prevalence and monitor annual trends to inform quality of care evaluations. Whether the purpose is to evaluate quality of care over time within the same institution or to make comparisons between institutions, we need to ensure that the data are sufficiently standardised and its quality is comparable across jurisdictions. Maintaining data quality is particularly salient, as the sensitivity, specificity and the NPV and PPV of the data sources impact their comparability of prevalence rates. Given the high predictive value, this data source could be used to screen for potential pressure ulcer cases, which can be verified and their causes identified through chart reviews. Furthermore, this process would generate a cohort of severe pressure ulcer cases for follow-up study.

We acknowledge that our study has some limitations. First, there are fundamental limitations when using administrative data for surveillance of conditions related to patient safety. Specifically, improved documentation can result in a higher frequency of reported cases, making it appear as though hospitals with a better documentation have worse performance. Similarly, Quan et 
Table 3 Stratifications of pressure ulcer cases in discharge abstract data and in nursing consultations

\begin{tabular}{|c|c|c|c|c|c|c|c|}
\hline \multirow{2}{*}{ Factors } & & \multicolumn{3}{|c|}{ Pressure ulcer coded in DAD } & \multicolumn{3}{|c|}{$\begin{array}{c}\text { Pressure ulcer found in } \\
\text { consultation }\end{array}$} \\
\hline & & Yes & No & OR & Yes & No & OR \\
\hline \multicolumn{8}{|c|}{ Definition 1 (7 ICD-10-CA codes) } \\
\hline \multirow[t]{3}{*}{ Age } & $18-34$ & 9 & 8658 & 1.00 & 15 & 51 & 1.00 \\
\hline & $35-64$ & 69 & 16712 & 3.97 & 150 & 410 & 1.24 \\
\hline & 65 and over & 85 & 13287 & 6.15 & 232 & 359 & 2.20 \\
\hline \multirow[t]{2}{*}{ Sex } & Male & 100 & 16624 & 1.00 & 221 & 437 & 1.00 \\
\hline & Female & 63 & 22033 & 0.48 & 176 & 383 & 0.91 \\
\hline \multirow[t]{5}{*}{ Number of comorbidities } & 0 & 0 & 5826 & & 2 & 52 & 1.00 \\
\hline & $1-3$ & 11 & 18401 & 1.00 & 44 & 241 & 4.75 \\
\hline & $4-6$ & 26 & 9457 & 2.31 & 92 & 210 & 11.39 \\
\hline & $7-9$ & 39 & 2976 & 21.92 & 87 & 126 & 17.95 \\
\hline & 10 or more & 87 & 1997 & 72.88 & 172 & 191 & 23.41 \\
\hline \multirow[t]{4}{*}{ Length of stay } & $0-30$ days & 57 & 36695 & 1.00 & 210 & 613 & 1.00 \\
\hline & $31-90$ days & 74 & 1650 & 28.87 & 128 & 162 & 2.31 \\
\hline & $91-180$ days & 22 & 236 & 60.01 & 37 & 30 & 3.60 \\
\hline & 181 days or more & 10 & 76 & 84.71 & 22 & 15 & 4.28 \\
\hline \multicolumn{8}{|c|}{ Definition 2 (29 ICD-10-CA codes) } \\
\hline \multirow[t]{3}{*}{ Age } & $18-34$ & 179 & 8488 & 1.00 & 15 & 51 & 1.00 \\
\hline & $35-64$ & 272 & 16509 & 0.78 & 150 & 410 & 1.24 \\
\hline & 65 and over & 219 & 13153 & 0.79 & 232 & 359 & 2.20 \\
\hline \multirow[t]{2}{*}{ Sex } & Male & 437 & 16287 & 1.00 & 221 & 437 & 1.00 \\
\hline & Female & 233 & 21863 & 0.40 & 176 & 383 & 0.91 \\
\hline \multirow[t]{5}{*}{ Number of comorbidities } & 0 & 0 & 5826 & & 2 & 52 & 1.00 \\
\hline & $1-3$ & 108 & 18304 & 1.00 & 44 & 241 & 4.75 \\
\hline & $4-6$ & 197 & 9286 & 3.60 & 92 & 210 & 11.39 \\
\hline & $7-9$ & 154 & 2861 & 9.12 & 87 & 126 & 17.95 \\
\hline & 10 or more & 211 & 1873 & 19.09 & 172 & 191 & 23.41 \\
\hline \multirow[t]{4}{*}{ Length of stay } & 0-30 days & 504 & 36248 & 1.00 & 210 & 613 & 1.00 \\
\hline & $31-90$ days & 117 & 1607 & 5.24 & 128 & 162 & 2.31 \\
\hline & $91-180$ days & 31 & 227 & 9.82 & 37 & 30 & 3.60 \\
\hline & 181 days or more & 18 & 68 & 19.04 & 22 & 15 & 4.28 \\
\hline
\end{tabular}

DAD, discharge abstract database; ICD-10-CA, International Disease Classification codes 10th version, Canada.

al drew a conclusion in a chart review study validating ICD-10 hospital discharge abstract data for the Agency for Healthcare Research and Quality patient safety indicators in hospital discharge abstract data. ${ }^{33}$ Due to findings of low sensitivity, they recommended that caution be used when rates of patient safety indicators are presented for 'quality of care reporting' as under-coded data generates falsely low patient safety indicator rates. Our study supports that administrative data are problematic when used for patient safety indicator surveillance. This illustrates the need for widespread improvements in both documentation and data abstraction of pressure ulcer occurrences for accurate data reporting.
Another limitation is that this study was conducted in one institution in an urban area over 1 year, thus it limiting generalizability, though this institution is one of the largest hospitals in Canada. An additional limitation is that we used ET documentation as the reference standard. Unfortunately, clear staging of pressure ulcers was not consistently included in the consultation notes. While ET nurses may be the most knowledgeable about identifying, and treating pressure ulcers, at this study's setting, they are most often consulted for the more severe pressure ulcer cases. As such, there is potential for bias as our study findings were less likely to include cases of stage I and II pressure ulcers, limiting its applicability to capture 


\begin{tabular}{|c|c|c|}
\hline Ulcer location & Number of cases & Prevalence \\
\hline Ankle & 14 & $1.1 \%$ \\
\hline Foot & 10 & $2.5 \%$ \\
\hline Heel & 42 & $10.6 \%$ \\
\hline Ischial & 4 & $1.0 \%$ \\
\hline Leg & 24 & $6.0 \%$ \\
\hline Sacral & 13 & $3.3 \%$ \\
\hline Trochanteric & 282 & $71.0 \%$ \\
\hline Other & 8 & $2.1 \%$ \\
\hline Total & 397 & $100.0 \%$ \\
\hline
\end{tabular}

all pressure ulcers. We recognise that not all hospitals may offer specialised wound care services like ET nurses, further limiting our study's generalisability.

There are several key practice implications from our study. One is that pressure ulcers are consistently coded using the ICD-10 L89.x codes and also codes for other skin injuries. Thus, there will be positive cases missed if only L89 codes are used, as evidenced by low sensitivity of definition 1. Second, more pressure ulcer cases would also be captured by improved documentation to clearly state the presence of a pressure ulcer versus just wound description. Third, coding specialists should be trained to use only L89 codes for pressure ulcers. Lastly, as recommended by Backman, documentation by nurses or other health professions should be considered valid sources for ICD coding to accurately capture hospital-related adverse events, such as pressure ulcers. ${ }^{4}$ Until these changes are made, a more inclusive case definition like definition 2 may be useful to identify pressure ulcer cases.

\section{FUTURE RESEARCH}

To proceed with quality improvement interventions, better methods are needed to quickly and accurately identify pressure ulcers in hospital charts and in coded data. Chart review and even the process of coding patient data are time-consuming and costly. Future research must include further validation of a case definition for pressure ulcer. Then, these validated cases can be used to develop algorithms for fast automated detection of pressure ulcer in electronic hospital chart data. Currently at our centre, a large $(n=3000)$ review of randomly selected hospital charts is underway to evaluate prevalence and validate ICD-10 codes for pressure ulcer in a larger sample. Next steps include using the validated terminology that describes pressure ulcer to develop autodetection algorithms. Autodetection, both in postdischarge and real-time admissions, will enable more effective tracking of pressure ulcer for the best possible treatment and quality improvement strategies for prevention. Ultimately, the same research methods could then be applied to other adverse events for fast accurate measurement.

\section{CONCLUSION}

This study provides a case definition for pressure ulcer surveillance in Canada using administrative health data. Our findings support the use of a more inclusive definition within administrative data for identifying pressure ulcers, specifically, the more severe ones (stage III, IV and unstageable) per National Pressure Ulcer Advisory Panel (NPUAP)'s staging system. It may be particularly useful for monitoring prevalence trends within a facility. However, low sensitivity of DAD for identification of pressure ulcer suggests that this data source may not be accurate for determining overall prevalence, and it should be used with caution if it is being compared with other prevalence studies.

Acknowledgements We would like to acknowledge the following individuals for their assistance with the study: Edie Attrell, Giuliana Bulloch, Nicole McKenzie, and Nicki Waters

Contributors $\mathrm{CH}$ designed the study and data acquisition methods, led analysis, interpreted the data and provided critical oversight of drafting and revising the manuscript. CH had final approval of the submission. JJ performed analysis and interpretation of the data. CE participated in interpretation of the data, drafting, revisions and submission of the manuscript. HW participated in drafting, critical appraisal and revision of the manuscript. BW performed the majority of data acquisition and participated in drafting, critical appraisal and revision of the manuscript. HQ contributed substantially to the conception and design of the study, the interpretation of data, critical appraisal and revision of the manuscript and final approval for submission.

Funding $\mathrm{JJ}$ received scholarship support from Ward of the 21st Century (W21C) - a partnership of the University of Calgary and Alberta Health Services. $\mathrm{HQ}$ received salary support from Alberta Innovates Health Solutions (AlHS) for this project.

Competing interests None declared.

Ethics approval The University of Calgary Health Research Ethics Board approved this study (File number: REB13-0211).

Provenance and peer review Not commissioned; externally peer reviewed.

Data sharing statement № additional data are available.

Open Access This is an Open Access article distributed in accordance with the Creative Commons Attribution Non Commercial (CC BY-NC 4.0) license, which permits others to distribute, remix, adapt, build upon this work non-commercially, and license their derivative works on different terms, provided the original work is properly cited and the use is non-commercial. See: http://creativecommons.org/ licenses/by-nc/4.0/

(C) Article author(s) (or their employer(s) unless otherwise stated in the text of the article) 2017. All rights reserved. No commercial use is permitted unless otherwise expressly granted.

\section{REFERENCES}

1. Redelings MD, Lee NE, Sorvillo F. Pressure ulcers: more lethal than we thought? Adv Skin Wound Care 2005;18:367-72.

2. Panel N. NPUAP pressure ulcer stages/Categories. http://www. npuap.org/resources/educational-and-clinical-resources/npuappressure-ulcer-stagescategories/.

3. Woodbury MG, Houghton PE. Prevalence of pressure ulcers in Canadian healthcare settings. Ostomy Wound Manage 2004;50:22-39.

4. Backman C, Vanderloo SE, Miller TB, et al. Comparing physical assessment with administrative data for detecting pressure ulcers in a large Canadian academic health sciences centre. BMJ Open 2016;6:e012490.

5. Cuddigan J, Berlowitz DR, Ayello EA. Pressure ulcers in America: prevalence, incidence, and implications for the future. Advances in Skin \& Wound Care 2001;14:208.

6. Tubaishat A, Papanikolaou P, Anthony D, et al. Pressure ulcers prevalence in the acute care setting: a systematic review, 2000-2015. Clin Nurs Res 2017;1:1-28. 
7. Dealey C, Posnett J, Walker A. The cost of pressure ulcers in the United Kingdom. J Wound Care 2012;21:261-6.

8. Brem H, Maggi J, Nierman D, et al. High cost of stage IV pressure ulcers. Am J Surg 2010;200:473-7.

9. Chan BC, Nanwa N, Mittmann N, et al. The average cost of pressure ulcer management in a community dwelling spinal cord injury population. Int Wound J 2013;10:431-40.

10. Smith IL, Nixon J, Brown S, et al. Pressure ulcer and wounds reporting in NHS hospitals in England part 1: Audit of monitoring systems. J Tissue Viability 2016;25:3-15.

11. Alderden J, Whitney JD, Taylor SM, et al. Risk profile characteristics associated with outcomes of hospital-acquired pressure ulcers: a retrospective review. Crit Care Nurse 2011;31:30-43.

12. Ash D. An exploration of the occurrence of pressure ulcers in a British spinal injuries unit. J Clin Nurs 2002;11:470-8.

13. Bergquist S, Frantz R. Pressure ulcers in community-based older adults receiving home health care. prevalence, incidence, and associated risk factors. Adv Wound Care 1999;12:339-51.

14. MCdS C, Caliri MHL, Hass VJ. Prevalência De úlceras de presão em pacientes críticos internados em um hospital Universitário. REME rev min enferm 2004:8:316-20.

15. Garber SL, Rintala DH. Pressure ulcers in veterans with spinal cord injury: a retrospective study. J Rehabil Res Dev 2003:40:433-42.

16. Gardiner JC, Reed PL, Bonner JD, et al. Incidence of hospitalacquired pressure ulcers - a population-based cohort study. Int Wound J 2016;13:809-20.

17. Hyun S, Li X, Vermillion B, et al. Body mass index and pressure ulcers: improved predictability of pressure ulcers in intensive care patients. Am J Crit Care 2014;23:494-501.

18. Jones KR, Fennie K. Factors influencing pressure ulcer healing in adults over 50: an exploratory study. J Am Med Dir Assoc 2007;8:378-87.

19. Lewis GM, Pham TN, Robinson E, et al. Pressure ulcers and risk assessment in severe burns. J Burn Care Res 2012;33:619-23.

20. New PW, Rawicki HB, Bailey MJ. Nontraumatic spinal cord injury rehabilitation: pressure ulcer patterns, prediction, and impact. Arch Phys Med Rehabil 2004;85:87-93.

21. Pedrosa IL, Silva MSML, Araújo AAde, et al. Pressure ulcers in elders and in non-elders: a historical cohort study. Online Brazilian Journal of Nursing 2014;13:82-91.

22. Rabadi MH, Vincent AS. Do vascular risk factors contribute to the prevalence of pressure ulcer in veterans with spinal cord injury? $J$ Spinal Cord Med 2011;34:46-51.

23. Schue RM, Langemo DK. Pressure ulcer prevalence and incidence and a modification of the Braden Scale for a rehabilitation unit. $J$ Wound Ostomy Continence Nurs 1998;25:36-43.

24. Spittle M, Collins RJ, Conner H. The incidence of pressure sores following lower limb amputations. Practical Diabetes International 2001;18:57-61.

25. Boettger JE. Effects of a pressure-reduction mattress and staff education on the incidence of nosocomial pressure ulcers. $J$ Wound Ostomy Continence Nurs 1997;24:19-25.

26. Cole L, Nesbitt C. A three year multiphase pressure ulcer prevalence/ incidence study in a regional referral hospital. Ostomy Wound Manage 2004;50:32-41.

27. Sheerin F, Gillick A, Doyle B. Pressure ulcers and spinal-cord injury: incidence among admissions to the Irish national specialist unit. $J$ Wound Care 2005;14:112-5.

28. Rennert R, Golinko M, Yan A, et al. Developing and evaluating outcomes of an evidence-based protocol for the treatment of osteomyelitis in Stage IV pressure ulcers: a literature and wound electronic medical record database review. Ostomy Wound Manage 2009;55:42.
29. Ham HW, Schoonhoven LL, Galer AA, et al. Cervical collar-related pressure ulcers in trauma patients in intensive care unit. J Trauma Nurs 2014;21:94-102.

30. Jin Y, Piao J, Lee SM. Evaluating the validity of the Braden scale using longitudinal electronic medical records. Res Nurs Health 2015;38:152-61.

31. Robitaille C, Dai S, Waters C, et al. Diagnosed hypertension in Canada: incidence, prevalence and associated mortality. CMAJ 2012;184:E49-E56.

32. Sadeghi B, White RH, Maynard G, et al. Improved coding of postoperative deep vein thrombosis and pulmonary embolism in administrative data (AHRQ Patient Safety Indicator 12) after introduction of new ICD-9-CM diagnosis codes. Med Care 2015;53:e37-e40.

33. Quan $\mathrm{H}$, Eastwood C, Cunningham CT, et al.Validity of AHRQ patient safety indicators derived from ICD-10 hospital discharge abstract data (chart review study). BMJ Open 2013;3:e003716.

34. Rosen AK, Itani KM, Cevasco M, et al. Validating the patient safety indicators in the Veterans Health Administration: do they accurately identify true safety events? Med Care 2012;50:74-85.

35. Borzecki AM, Kaafarani HM, Utter GH, et al. How valid is the AHRQ Patient Safety Indicator 'postoperative respiratory failure'? J Am Coll Surg 2011;212:935-45.

36. Chen $\mathrm{Q}$, Rosen AK, Cevasco M, et al. Detecting patient safety indicators: how valid is 'foreign body left during procedure' in the Veterans Health Administration? J Am Coll Surg 2011;212:977-83.

37. Koch CG, Li L, Hixson E, et al. What are the real rates of postoperative complications: elucidating inconsistencies between administrative and clinical data sources. J Am Coll Surg 2012;214:798-805

38. Lyder $\mathrm{CH}$, Wang $\mathrm{Y}$, Metersky $\mathrm{M}$, et al. Hospital-acquired pressure ulcers: results from the national Medicare Patient Safety monitoring System study. J Am Geriatr Soc 2012;60:1603-8.

39. Fogerty M, Guy J, Barbul A, et al. African Americans show increased risk for pressure ulcers: a retrospective analysis of acute care hospitals in America. Wound Repair Regen 2009;17:678-84.

40. Kröger K, Niebel W, Maier I, et al. Prevalence of pressure ulcers in hospitalized patients in Germany in 2005: data from the Federal Statistical Office. Gerontology 2009;55:281-7.

41. Ackroyd-Stolarz S, Bowles SK, Giffin L. Validating administrative data for the detection of adverse events in older hospitalized patients. Drug Healthc Patient Saf 2014;6:101.

42. Polancich S, Restrepo E, Prosser J. Cautious use of administrative data for decubitus ulcer outcome reporting. Am J Med Qual 2006;21:262-8.

43. Meddings JA, Reichert $\mathrm{H}$, Hofer $\mathrm{T}$, et al. Hospital report cards for hospital-acquired pressure ulcers: how good are the grades? Ann Intern Med 2013;159:505-13.

44. Berlowitz DR, Brandeis GH, Brand HK, et al. Evaluating pressure ulcer occurrence in long-term care: pitfalls in interpreting administrative data. J Clin Epidemiol 1996;49:289-92.

45. Barczak CA, Barnett RI, Childs EJ, et al. Fourth national pressure ulcer prevalence survey. Adv Wound Care 1997:10:18-32.

46. VanGilder C, Amlung S, Harrison P, et al. Results of the 20082009 International Pressure Ulcer Prevalence ${ }^{\mathrm{TM}}$ Survey and a 3-year, acute care, unit-specific analysis. Ostomy Wound Manage 2009;55:39-45

47. Quan H, Khan N, Hemmelgarn BR, et al.Validation of a case definition to define hypertension using administrative data. Hypertension 2009;54:1423-8.

48. Chen $\mathrm{G}$, Khan N, Walker R, et al. Validating ICD coding algorithms for diabetes mellitus from administrative data. Diabetes Res Clin Pract 2010;89:189-95. 